\title{
Supporting Information for: \\ Low-Frequency (Gigahertz to Terahertz) Depolarized Raman Scattering off $n$-Alkanes, Cycloalkanes, and Six-Membered Rings: a Physical Interpretation
}

Andrew J. Farrell, Mario González Jiménez, Gopakumar Ramakrishnan, and Klaas Wynne

School of Chemistry, University of Glasgow, UK

Containing 14 pages, 8 figures, and 6 tables.

1. UNCERTAINTIES IN THE OKE SPECTRA 2

2. AMPLITUDE OF THE OKE SIGNAL 2

2.1. Molarity of n-alkanes $\quad 2$

2.2. Librations $\quad 4$

2.2.1. The moment of inertia as a function of alkyl chain length 5

2.2.2. The dependence of the librational frequency on alkyl chain length 5

2.2.3. The dependence of the amplitude of librations on alkyl chain length 6

2.3. The fast- $\beta$ process (cage rattling) 6

2.3.1. The dependence of the fast- $\beta$ process frequency on alkyl chain length 6

2.3.2. The dependence of the amplitude of fast- $\beta$ process on alkyl chain length 7

2.4. Estimation of the timescales for diffusive processes 8

2.4.1. Viscosities and densities 8

2.5. $\alpha$-relaxation (orientational diffusion) 9

2.5.1. The $\alpha$-relaxation timescale 9

2.5.2. Spheroid slip conditions 10

2.5.3. The $\alpha$-relaxation amplitude 10

2.6. $\beta$-relaxation (translational diffusion) 11

2.7. Molecular Geometries 11

3. FIT PARAMETERS

4. REFERENCES 


\section{UNCERTAINTIES IN THE OKE SPECTRA}

Because OKE data are acquired in the time domain, uncertainties in the Fourier transformed frequency domain OKE spectrum are non-trivial. Noise manifests in the frequency domain as high frequency artefacts that generally interfere with the spectrum only above $\sim 20 \mathrm{THz}$. One should also note the uncertainties introduced by small day to day changes in the laser pulse profile caused by a variety of factors such as laser crystal/optics cleanliness and changes in atmospheric conditions; however, this too has little influence at intermolecular dynamical frequencies.

For the frequency domain data fitted with up to 4 lineshapes, the greatest source of uncertainty comes from excess degrees of freedom. The modes used to model small molecule spectra often have a high degree of overlap and interdependence (e.g., pentane, cyclohexane, and propane at $\geq 170 \mathrm{~K}$ ), and can only be arrived at through iteration and extrapolation of the trends observed in one or more different series of experiments. Although this work represents just one of many possible fits for each individual spectrum, and many can be fit with fewer lineshapes, these are the only solutions that were found to produce a consistent and physical interpretation across all series. So, despite the often-large uncertainties in individual fitting parameter values, we argue that these fits are compelling in a collective context instead.

\section{AMPLITUDE OF THE OKE SIGNAL}

The OKE data presented in this work are all taken under comparable circumstances (comparable laser power, pulse width, and focusing conditions). Therefore, the amplitudes are (within a degree of error) comparable between the different samples studied. A key aspect of this paper is the investigation of the amplitude of the four main components of the OKE spectra $(\alpha-$ relaxation, $\beta$-relaxation, the fast $\beta$ process, and librations) as a function of alkyl chain length. Therefore, expressions are required for the absolute amplitude of OKE signals.

The heterodyne-detected OKE signal in the time-domain is given by the expression ${ }^{1}$

$$
S_{\text {OKE }}(\tau)=M \int_{-\infty}^{\infty} d t I_{\text {probe }}(t-\tau) \int_{0}^{\infty} d t_{1} I_{\text {pump }}\left(t-t_{1}\right) R_{i j k l}^{(3)}\left(t_{1}\right),
$$

where $I_{\text {pump }}$ and $I_{\text {probe }}$ are the pump and probe laser intensity profiles, and the third-order susceptibility is given by ${ }^{2}$

$$
R_{i j k l}^{(3)}(\tau)=\frac{i}{\hbar}\left\langle\left[\Pi_{i j}(\tau), \Pi_{k l}(0)\right]_{-}\right\rangle
$$

where $\Pi$ is the polarizability tensor, which has contributions from the molecular polarizability as well as interaction-induced contributions, and $i j k l$ label the polarizations of the four fields involved. Since the third-order susceptibility is written in terms of the molecular polarizability etc., the overall signal in Eq. (SI) has to be scaled with the molarity $M$ of the sample. In the absence of interaction-induced effects, the third-order susceptibility becomes

$$
R_{i j k l}^{(3)}(\tau)=\frac{i}{\hbar}\left\langle[\beta(\tau), \beta(0)]_{-}\right\rangle
$$

where $\beta$ is the anisotropic molecular polarizability, and we will use this form below as a shorthand for the full expression including interaction-induced effects.

The molecules studied in this work are of reduced dimensionality. If we consider how the geometry of the $n$-alkanes varies with the number of carbons, $n$, it is reasonable to begin by approximating a linear relationship between chain length $L$ and $n$, but it is of course $a s$ true that the surface area and volume of the molecule will also vary with $n$. For this reason, it is useful to consider the intermolecular interactions in terms of $n$ as opposed to more intuitive properties like the molecular surface area, and thanks to the simplicity of these molecules we can break down the intermolecular interactions into the sum of (essentially) interatomic components. 


\subsection{Molarity of $n$-alkanes}

Table S1. Molarity of n-alkanes, cycloalkanes and six-membered rings from their density.

\begin{tabular}{|c|c|c|c|}
\hline $\begin{array}{l}n \text {-Alkane } \\
\mathrm{C}_{\mathrm{n}} \mathrm{H}_{2 \mathrm{n}+2} \\
\end{array}$ & $\begin{array}{c}\text { Density }[\mathrm{g} / \mathrm{L}] \\
\left(\text { at } 20^{\circ} \mathrm{C}\right)\end{array}$ & $\begin{array}{c}\text { Mol. weight } \\
(\mathrm{g} / \mathrm{mol})\end{array}$ & $\begin{array}{l}\text { Molarity } \\
(\mathrm{mol} / \mathrm{L})\end{array}$ \\
\hline 5 & 626 & 72 & 8.694 \\
\hline 6 & 659 & 86 & 7.663 \\
\hline 7 & 684 & 100 & 6.84 \\
\hline 8 & 703 & 114 & 6.167 \\
\hline 9 & 718 & 128 & 5.609 \\
\hline 10 & 730 & 142 & 5.141 \\
\hline 11 & 740 & 156 & 4.744 \\
\hline 12 & 749 & 170 & 4.406 \\
\hline 13 & 756 & 184 & 4.109 \\
\hline 14 & 763 & 198 & 3.854 \\
\hline 15 & 769 & 212 & 3.627 \\
\hline 16 & 773 & 226 & 3.420 \\
\hline 17 & 777 & 240 & 3.238 \\
\hline 20 & 789 & 254 & 3.106 \\
\hline $\begin{array}{c}\text { Cycloalkane } \\
\mathrm{C}_{\mathrm{n}} \mathrm{H}_{2 \mathrm{n}}\end{array}$ & $\begin{array}{c}\text { Density }[\mathrm{g} / \mathrm{L}] \\
\left(\text { at } 20^{\circ} \mathrm{C}\right)\end{array}$ & $\begin{array}{l}\text { Mol. weight } \\
(\mathrm{g} / \mathrm{mol})\end{array}$ & $\begin{array}{l}\text { Molarity } \\
(\mathrm{mol} / \mathrm{L})\end{array}$ \\
\hline 5 & 751 & 70 & 10.73 \\
\hline 6 & 779 & 84 & 9.274 \\
\hline 7 & 811 & 98 & 8.276 \\
\hline 8 & 834 & 112 & 7.446 \\
\hline 10 & 871 & 140 & 6.221 \\
\hline Cyclohexene & 811 & 82 & 9.890 \\
\hline 1,4-Cyclohexadiene & 847 & 80 & 10.59 \\
\hline Benzene & 876 & 78 & 11.23 \\
\hline
\end{tabular}




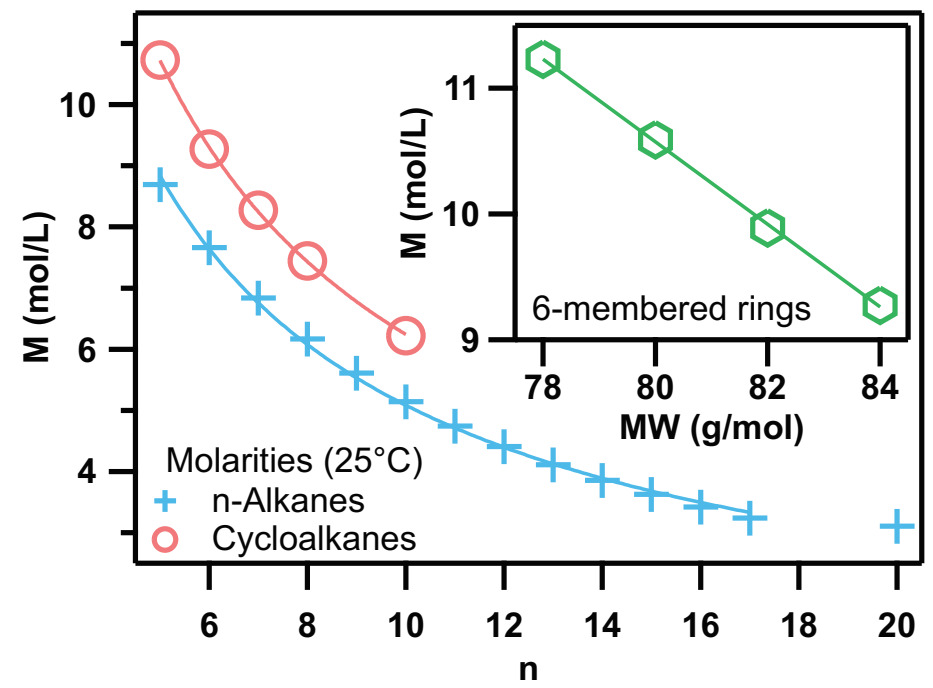

Figure S1. Molarity of n-alkanes, cycloalkanes, and 6-membered rings at room temperature. The solid lines (main) are power-law fits with exponents of -0.79 and -0.78 for n-alkanes and cycloalkanes respectively. Six-membered rings are fit with a linear trend with molecular weight (inset). $80 \mathrm{~g} / \mathrm{mol}$ ring is 1,4 -cyclohexadiene.

The molarities of the $n$-alkanes and cycloalkanes are proportional to $\mathrm{n}^{-0.79}$ and $\mathrm{n}^{-0.78}$ respectively, while the molarity of the 6 membered rings is directly proportional to the molecular weight.

\subsection{Librations}

First the amplitude, frequency, and alkyl chain length dependent amplitude of the librations will be considered. Librations are strictly speaking collective motions but, of all the low-frequency motions and relaxations, are least susceptible to collective effects. The librational motion will be described as a harmonic motion of the angle $q$ of the molecule. The third-order susceptibility can then be written as ${ }^{\mathrm{I}}$

$$
R_{i j k l}^{(3)}(\tau)=\beta^{2} \frac{i}{\hbar}\left\langle[\theta(\tau), \theta(0)]_{-}\right\rangle=\frac{\beta^{2}}{I \Omega} \sin (\Omega t) e^{-\gamma t / 2}
$$

where $\gamma$ is the damping rate, $\omega_{0}$ the librational angular frequency, $I$ is the moment of inertia, and

$$
\Omega \equiv \sqrt{\omega_{0}^{2}-\gamma^{2} / 4} .
$$

Under the simplifying assumption of infinitely short laser pulses, the OKE signal then becomes

$$
S_{\text {OKE }}(\tau)=M I_{\text {laser }}^{2} \frac{\beta^{2}}{I \Omega} \sin (\Omega t) e^{-\gamma t / 2},
$$

where $I_{\text {laser }}$ is proportional to the laser intensity.

Librations appear to increase in frequency from pentane to octane and saturate at the same frequency for longer alkanes, which is not intuitive. In the rigid rod regime, where we can reasonably speak in terms of librations, one might expect an increasing moment of inertia for longer chains to result in a redshift, as with the orientational diffusion. On the contrary we can show mathematically that the librational frequency should actually increase. We can work out the total repulsive restoring force as the summation of individual restoring forces. A librating rigid rod is illustrated in Figure $S_{2}$. 


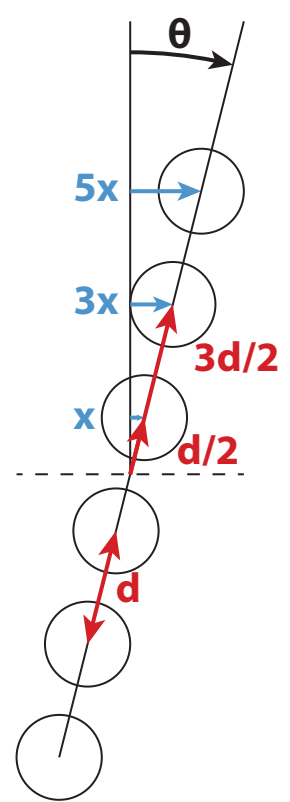

Figure S2. Cartoon of libration in various chain lengths, with the radial distance of each unit marked (using the small angle approximation).

\subsubsection{The moment of inertia as a function of alkyl chain length}

We will now consider the dependence of the moment of inertia $I_{n}$ on alkyl chain length $n$. Referring to the definitions in Figure S2, we obtain

$$
\begin{aligned}
& I_{2}=2 m\left(\frac{d}{2}\right)^{2}=2 m d^{2} \frac{1}{4} \\
& I_{4}=2 m d^{2}\left[\left(\frac{1}{2}\right)^{2}+\left(\frac{3}{2}\right)^{2}\right]=2 m d^{2} \frac{10}{4}, \\
& I_{6}=2 m d^{2}\left[\frac{10}{4}+\left(\frac{5}{2}\right)^{2}\right]=2 m d^{2} \frac{35}{4}
\end{aligned}
$$

where $m$ is the mass of a $\mathrm{CH}_{2}$ unit and $d$ is the $\mathrm{C}-\mathrm{C}$ bond length, so for any $n$

$$
I_{n}=2 m d^{2}\left[\sum_{i=2,4,6,8, \ldots}^{n}\left(\frac{i-1}{2}\right)^{2}\right]=2 m d^{2} \frac{1}{24}\left(n^{2}-1\right) n
$$

\subsubsection{The dependence of the librational frequency on alkyl chain length}

For simplicity we will consider how the restoring torque, $T$, varies with chain length of the alkanes when $n$ is even. The magnitude of the torque is, of course, the perpendicular force times distance from the center of rotation. Therefore, using the small angle approximation, the restoring torque on each $\mathrm{CH}_{2}$ unit is given by Hooke's law 


$$
\begin{aligned}
T_{2} & =-2 k x \frac{d}{2}=-2 k\left(\frac{d}{2}\right)^{2} \sin \theta \cong-\frac{1}{2} k d^{2} \theta \\
T_{4} & =-2 k\left(x \frac{d}{2}+3 x \frac{3 d}{2}\right) \\
T_{n} & =-2 k\left(x \frac{d}{2}+3 x \frac{3 d}{2}+5 x \frac{5 d}{2}+\cdots\right)=-2 k \frac{d}{2}\left(1+3^{2}+5^{2}+\cdots\right) x \\
& \cong-2 k\left(\frac{d}{2}\right)^{2}\left(1+3^{2}+5^{2}+\cdots\right) \theta=-2 k \theta\left(\frac{d}{2}\right)^{2} \sum_{i=2,4,6, \ldots}^{n}(i-1)^{2} \\
& =-2 k \theta\left(\frac{d}{2}\right)^{2} \frac{1}{6} n\left(n^{2}-1\right)=-\frac{1}{12} k d^{2} \theta n\left(n^{2}-1\right)
\end{aligned}
$$

where, $\theta$ is the angle of libration, $x$ is the displacement of the first (inner) $\mathrm{CH}_{2}$ unit, $k$ is Hooke's constant for each $\mathrm{CH}_{2}$ unit, and a factor of 2 arises through symmetry. The relationship between the restoring torque and $n$ is therefore

$$
T_{n}=-k_{\text {effective }} \theta \propto n\left(n^{2}-1\right)
$$

where

$$
k_{\text {effective }}=\frac{1}{12} k d^{2} n\left(n^{2}-1\right)
$$

Thus, the librational frequency depends on $n$ as

$$
\omega_{0}=\sqrt{\frac{k_{\text {effective }}}{I}} \propto \sqrt{\frac{n\left(n^{2}-1\right)}{\left(n^{2}-1\right) n}} \sim \text { const. }
$$

This relationship fits all the data except for a small deviation for the shortest molecules. This is to be expected since this theory is dependent on both rigidity and the strength of solvent-molecule interactions varying with $n$ (in other words, the surface area of the molecule varying with $n$ ).

\subsubsection{The dependence of the amplitude of librations on alkyl chain length}

We can now work out the strength of the OKE signal due to librations. Using Eq. (S6), assuming the damping is weak and can be ignored

$$
S_{\text {OKE }}=M I_{\text {laser }}^{2} \frac{\beta^{2}}{I \omega_{0}} \propto n^{-0.5} \frac{n^{2}}{n\left(n^{2}-1\right)} \cong n^{-1.5},
$$

where $\beta \propto n$ has been assumed for linear alkanes. This result does not correspond well with the experimental data, which show that the librational amplitude is approximately constant with alkyl chain length. However, the above equation for the amplitude does not include the effect of collision-induced enhancement of the molecular polarizability, which must also scale with $n$ or a higher power thereof.

\subsection{The fast- $\beta$ process (cage rattling)}

\subsubsection{The dependence of the fast- $\beta$ process frequency on alkyl chain length}

Figure $\mathrm{S}_{3}$ illustrates the time-average intermolecular potential about a single alkane molecule, approximated by a linear chain of $\mathrm{CH}_{\mathrm{n}}$ spheres. The fast- $\beta$ process frequency appears to stay roughly constant for short chains, eventually drops off with longer chains. We should be able to explain the trend for short chains if we assume rigidity, whereas longer chains will become progressively floppier. Since the fast- $\beta$ mode is collision-induced, collisions orthogonal to the molecular axis should produce the greatest collision-induced anisotropy. 


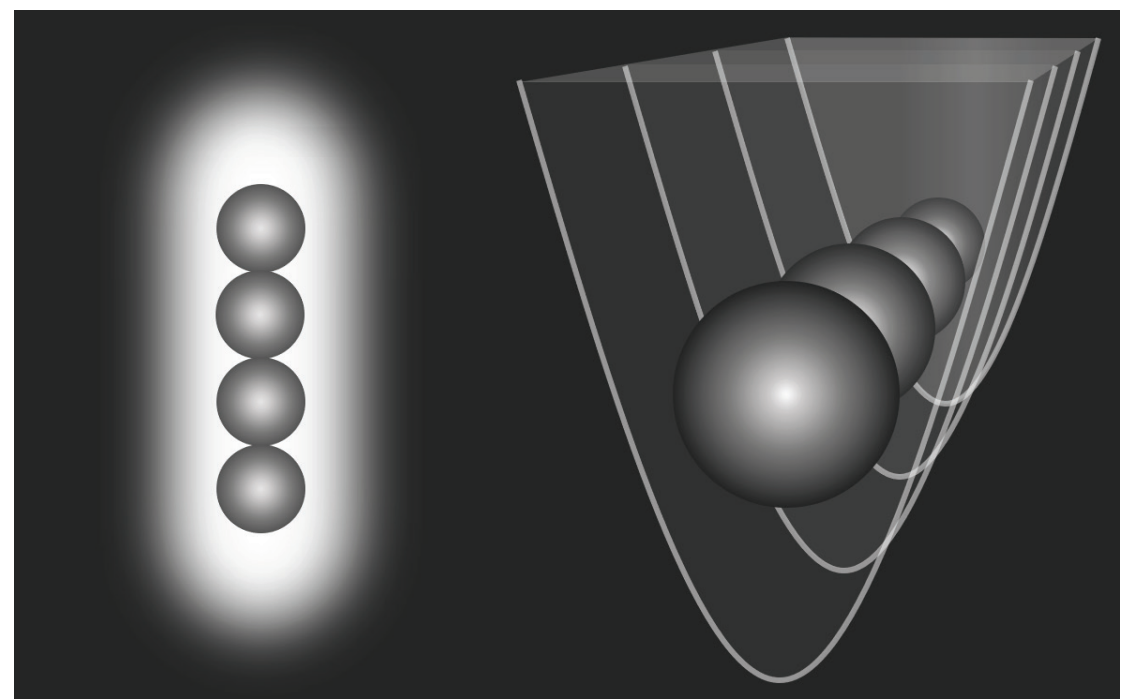

Figure $S_{3}$. Cartoons of the time-averaged intermolecular potential landscape of the $\boldsymbol{n}$-alkanes (left) and the discretised one-dimensional harmonic potentials about each $\mathrm{CH}_{\mathrm{n}}$ unit (right).

Assuming a harmonic potential, the intermolecular force, $F_{n}$ felt by the molecule during a broadside translation can be defined simply as the sum of interatomic forces

$$
F_{n}=-n k x
$$

where $k$ is the force constant and $x$ is the displacement. Since $\omega_{0}=\sqrt{k / m}$, where $\omega_{0}$ is the angular frequency of the harmonic oscillator, then we find that

$$
\omega_{0} \propto \sqrt{\frac{n}{n}}=\text { const }
$$

The frequency of the fast- $\beta$ mode is expected to be independent on chain length or ring size. This does indeed match the experimental data for the small, rigid alkanes and the small 'uncrumpled' rings. When it comes to the 'crumpled' cyclooctane (Figure $\mathrm{S}_{4}$ ) onwards, we would expect this relation to break down. The simple intermolecular interaction outlined in Figure $\mathrm{S}_{3}$ relies on each atomic unit participating in collisions. As molecules become more spherical, the shielded mass still acts as an inertial load, but its contribution to the spring constant, $k$ diminishes. Simply put, the $m \propto n$ relationship still holds, while $k \propto n$ does not.

Figure $S_{4}$. Side view of boat-chair cyclooctane.

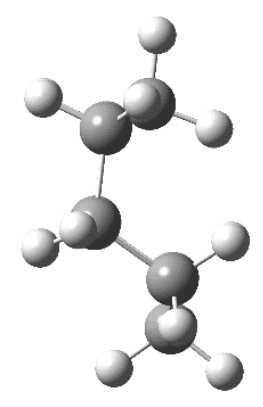

\subsubsection{The dependence of the amplitude of fast- $\beta$ process on alkyl chain length}

In a Maxwell-Boltzmann distribution, the most probable velocity, $v$, is given as

$$
v^{2}=\frac{2 k_{B} T}{m}
$$

where $k_{B}$ is the Boltzmann constant, $T$ is the temperature and $m$ is the mass of the molecule, which scales with $n$. Next consider that each $\mathrm{CH}_{2}$ unit has the same polarizability, and broadside collisions of long chains and short chains have different velocities. It follows that the $\mathrm{CH}_{2}$ units in shorter chains with greater velocities more closely approach their nearest neighbors, and therefore yield a greater collision-induced signal. We must also note that the polarizability per unit volume should be influenced 
mostly by the number density (or $\mathrm{CH}_{\mathrm{n}}$ unit density) of the liquid which only increases by $25 \%$ over the pentane to hexadecane (3-fold increase) range.

If the intermolecular repulsion falls off exponentially as

$$
V(r)=V_{0} \exp \left(-\frac{r}{r_{0}}\right)
$$

where $r_{\mathrm{o}}$ is close to the Bohr radius and ${ }^{3} V_{\mathrm{o}}$ is $\sim 10^{-17} \mathrm{~J}$ and considering collisions at the most probable energy, will lead to a minimum collision distance of

$$
V_{0} \exp \left(-\frac{r}{r_{0}}\right)=\frac{1}{2} m v^{2} \rightarrow r=-r_{0} \ln \left(\frac{m v^{2}}{2 V_{0}}\right) .
$$

In the dipole-induced-dipole approximation, the interaction-induced term in Raman scattering has the form ${ }^{2}$

$$
\Pi^{I I}=\sum \alpha \cdot \boldsymbol{T} \cdot \alpha,
$$

where $\alpha$ is the molecular polarizability and $\boldsymbol{T}$ is the dipole interaction tensor given by

$$
\boldsymbol{T}=\frac{3 \vec{r} \vec{r}-\boldsymbol{I}}{r^{3}},
$$

where $\overrightarrow{\boldsymbol{r}}$ is the unit vector connecting the centers of mass of both molecules. Given that the laser measures a constant volume throughout, one should consider the changing material density. Fortunately, the density changes can be accounted for by scaling the time domain signals so that they have the same electronic response amplitude. We are therefore measuring a constant mass of sample to a reasonable approximation.

The strength of the OKE signal scales with $\Pi^{2}$, and therefore as $r^{-6}$, this gives using Eq. (SI8)

$$
S_{C I} \propto \ln ^{-6}\left(\frac{m v^{2}}{2 V_{0}}\right)
$$

If we refer to a single $\mathrm{CH}_{2}$ unit, this becomes

$$
S_{C I} \propto \ln ^{-6}\left(\frac{14 \mathrm{a} v^{2}}{2 V_{0}}\right),
$$

where $a$ is the atomic mass unit. Using the velocity of the entire molecule as the velocity of the $\mathrm{CH}_{2}$ unit, we find

$$
S_{C I} \propto \ln ^{-6}\left(\frac{7 k_{B} T}{V_{0}(7 n+C)}\right)
$$

where $n$ is the number of carbons and $C$ is a constant with value 1 for alkanes (accounting for terminal hydrogens) or o for cycloalkanes.

\subsection{Estimation of the timescales for diffusive processes}

\subsubsection{Viscosities and densities}

In order to model diffusive lifetimes as a function of temperature or chain length, the viscosities must first be modelled. Dynamic viscosity $(\eta)$ data were obtained from the literature for alkanes ${ }^{4,5}$ and cycloalkanes ${ }^{6}$.

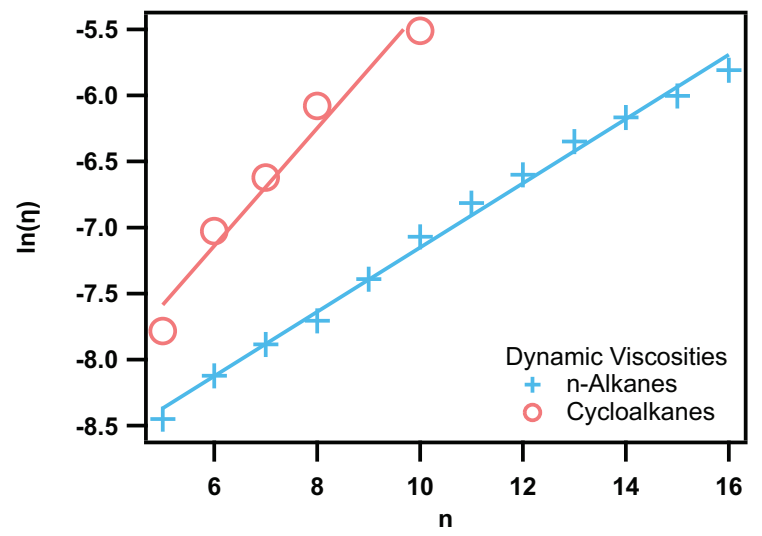

Figure $S_{5}$. Viscosities of $\boldsymbol{n}$-alkanes $(+)$ and cycloalkanes $(\circ)$ as a function of carbon chain length. 
With the results shown in Figure $\mathrm{S}_{5}$, the dynamic viscosities were fit to the simple relationships

$$
\begin{aligned}
& \ln \left(\eta_{\text {alk }}\right)=0.24 n-9.59 \\
& \ln \left(\eta_{\text {cyc }}\right)=0.45 n-9.81
\end{aligned}
$$

Likewise, the OKE spectrum of liquid propane depends on the dynamic viscosity, which needed to be modeled as a function of temperature. Additionally, the librational and fast- $\beta$ contributions to OKE spectrum are dependent on the change in density. Data for the viscosity and density $(\rho)$ of propane as a function of temperature ${ }^{4}$ are shown in Figure S6.

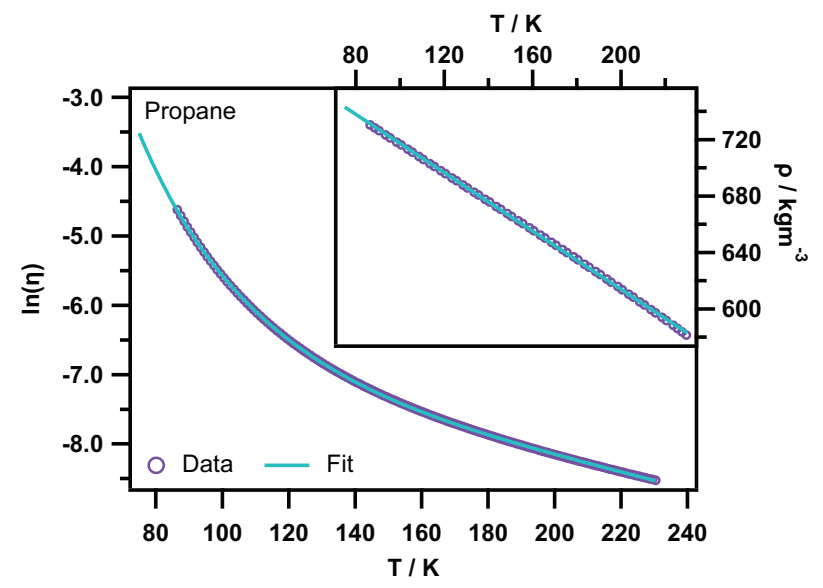

Figure S6. Fits of viscosity (main) and density (inset) of liquid propane as a function of temperature. Data ( $\circ$ ) retrieved from ref.4 were modeled and extrapolated into the supercooled regime.

These data were modeled with the relations

$$
\begin{gathered}
\ln (\eta)=-16.1+782 T^{-1}+3.44 \cdot 10^{-2} T-6.99 \cdot 10^{-5} T^{2} \\
\rho=-1.03 n+821
\end{gathered}
$$

\section{5. a-relaxation (orientational diffusion)}

\subsubsection{The $\alpha$-relaxation timescale}

The Stokes-Einstein equation relates the diffusion rate $D$ to the thermal energy and a friction factor, $\zeta$ specific to the material as ${ }^{7}$

$$
D=\frac{k_{B} T}{\zeta} \text {. }
$$

Typically, we can estimate the orientational diffusion time constant, $D_{r}$ using the Stokes-Einstein-Debye relation for spheres, where the friction factor is given as ${ }^{8}$

$$
\zeta_{r}=6 \eta V
$$

where $\eta$ is the dynamic viscosity and $V$ is the molecular volume. Given the rod-like geometry of the $n$-alkanes, we should also consider the friction factors, which have been derived for rods. It is the out-of-plane tumbling rotations that will contribute the greatest OKE signal in this work, as opposed to the, essentially frictionless, spinning about a rod's major axis. The friction factor for out-of-plane tumbling is given by ${ }^{9-I I}$

$$
\zeta_{r}=\frac{\pi \eta L^{3}}{3\left[\ln \left(\frac{2 L}{d}\right)-0.5\right]}
$$

where $L$ and $d$ are the length and diameter of the rod. These are the friction factors for perfect 'stick' diffusion but can be used to calculate the 'slip' diffusion by applying an aspect ratio dependent correction factor calculated in ref. ${ }^{12}$ and shown in Figure $\mathrm{S} 7$.

OKE is a four-wave mixing technique, meaning the orientational diffusion lifetime observed experimentally is related to the diffusion rate through the relation ${ }^{7}$

$$
\tau_{r}=\frac{1}{6 D_{r}} .
$$




\subsubsection{Spheroid slip conditions}

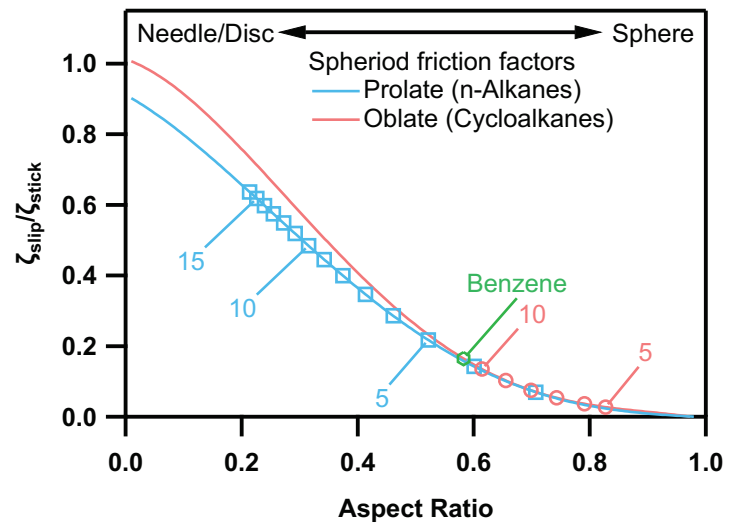

Figure $S_{7}$. Slip to stick friction factors as a function of aspect ratio from ref. ${ }^{\mathrm{I2}}$. The positions of the $n$-alkanes $($ blue $\square)$, cycloalkanes $($ red $\circ)$ and benzene (green $\circ$ ) marked.

'Slip' condition friction factors have been derived for spheroids of various aspect ratios. This is useful to this work as the geometry of the $n$-alkanes and cycloalkanes can be approximated by prolate and oblate spheroids respectively. Fitting these friction factor data enables us to find each alkane's corresponding slip to stick ratio and hence calculate the orientational diffusion lifetime. It is not intuitive from Figure $\mathrm{S} 7$ but $\mathrm{Hu}$ and Zwanzig note that prolate friction ratios rapidly approach 1 for very small aspect ratios. This is not represented here.

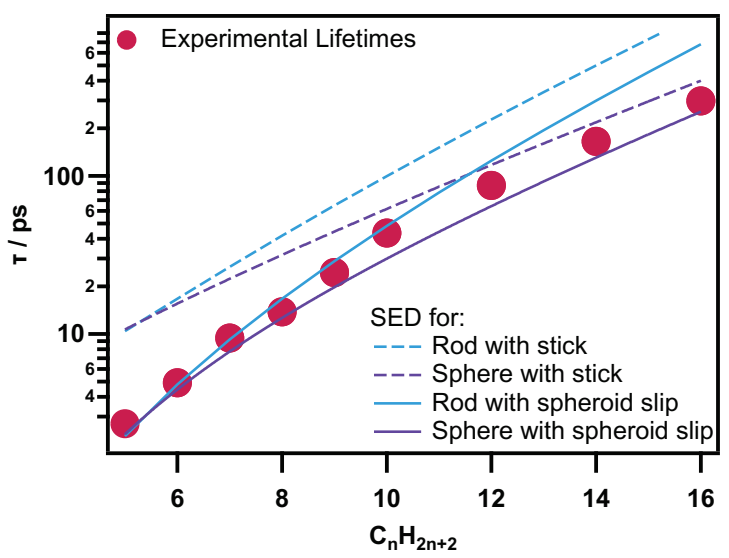

Figure S8. Comparison of SED for slip and stick boundary conditions with experimental $\alpha$-relaxation lifetimes $(\bullet)$ of $\boldsymbol{n}$-alkanes. 'Stick' (dashed lines) and 'slip' (solid lines) conditions are shown for both rods (blue lines) and spheres (purple lines).

Lifetimes for slip boundary conditions for both rods and spheres were found using the friction factors calculated for various spheroid aspect ratios. The slip conditions yield the best results for shorter chains, and where rod-like orientational diffusion is in closer agreement with experiment up to $c a$. decane. Longer chains diffuse faster than theory probably due to the increased significance of gauche conformers producing a shift from rod-like to spherical. This is reflected in Figure S8 as longer chains display orientational diffusion lifetimes closer to that of a sphere, regardless of slip/stick condition. We should also note that the difference between rod and sphere SED lifetimes are negligible at pentane, and as Figure S7 shows, the cyclic molecules (and pentane) all have aspect ratios closer to spherical. We argue that it is therefore unnecessary to apply any prolate/oblate (rod/disc)-SED equations to any other molecules in this work- the spheroid friction factors alone are sufficient.

\subsubsection{The $\alpha$-relaxation amplitude}

The pure rotational ( $\alpha$-relaxation) component of the OKE signal scales as

$$
S_{O K E} \propto M \beta^{2}
$$

and therefore 


$$
S_{O K E} \propto n^{1.21}
$$

for linear alkanes and

$$
S_{O K E} \propto n^{3.22}
$$

for cycloalkanes.

\section{6. $\beta$-relaxation (translational diffusion)}

$\beta$-relaxation describes molecular translational diffusion for which we apply the Stokes-Einstein relation that has a friction factor of ${ }^{13}$

$$
\zeta_{t}=C \pi \eta r
$$

where $r$ is the hydrodynamics radius and $C$ is a constant that can take on values between 4 and 6 pertaining to perfect slip and perfect stick conditions respectively. In this work with small weakly-interacting hydrocarbons, we apply the slip condition throughout.

For consistency, we should again consider the rod-like geometry of the $n$-alkanes and its effect on translational diffusion. The translational friction factor for a rod is given $b^{10}$

$$
\zeta_{t}=\frac{3 \pi \eta L}{\ln \left(\frac{2 L}{d}\right)}
$$

Translational diffusion produces a normal distribution of molecular displacements after some time, $t$. The variance of the distribution is given by

$$
\sigma^{2}=6 D_{t} t
$$

$\beta$-relaxation is also an interaction-induced process and therefore the $\beta$-relaxation amplitude should scale like the fast- $\beta$ process. This appears to be borne out by the data presented here.

\subsection{Molecular Geometries}

The minor radius of the rod should consider both minor axes of the alkane, which will be slightly different due to C-C bond projections. One of the minor radii, $r_{c}$, will be equivalent to the radius of methane, $\mathrm{r}_{\mathrm{CH}_{4}}$ is approximately the sum of the $\mathrm{C}-\mathrm{H}$ bond length and van der Waals radius:

$$
r_{c} \equiv r_{C H_{4}}=1.09+1.2=2.29 \AA
$$

The other minor radius, $r_{b}$ will be longer purely due to the additional $\mathrm{C}-\mathrm{C}$ bond component

$$
r_{b}=r_{\mathrm{CH}_{4}}+\frac{1.54 \sin \left(35^{\circ}\right)}{2}=2.73 \AA
$$

The effective minor axis radius, $r_{m}$ that conserves the molecular volume is therefore

$$
r_{m}=\sqrt{r_{c} r_{b}}=2.50 \AA
$$

The rod length is calculated as

$$
L=\left(2 r_{C H_{4}}+1.26(n-1)\right) \AA
$$

where $\mathrm{n}$ is the number of carbons.

For the non-alkanes, but including propane, the hydrodynamic radius was taken for a sphere of equivalent volume, calculated using the liquid densities scaled by Avogadro's number $\mathrm{N}_{\mathrm{A}}$.

$$
\mathrm{r}=\left(\frac{3}{4 \pi} \frac{1000 \rho}{M N_{A}}\right)^{\frac{1}{3}}
$$

where the density, $\rho$ is in units of $\mathrm{kg} / \mathrm{m}^{3}$ and $\mathrm{M}$ is the molecular mass in $\mathrm{g} / \mathrm{mol}$. 


\section{FIT PARAMETERS}

Table S2. Fit parameters for the $\boldsymbol{n}$-alkanes at $25^{\circ} \mathbf{C} . \Omega_{\mathrm{I}}$ are fixed values; $\alpha$-relaxation $\Omega_{\mathrm{I}}$ is fixed to the libration $\omega_{\mathrm{o}}$ and $\beta$-relaxation $\Omega_{\mathrm{I}}$ is fixed to the fast- $\beta \omega_{\circ}$.

\begin{tabular}{|c|c|c|c|c|c|c|c|c|c|c|c|c|}
\hline & \multicolumn{3}{|c|}{$\alpha$-relaxation } & \multicolumn{3}{|c|}{$\beta$-relaxation } & \multicolumn{3}{|c|}{ Fast- $\beta$} & \multicolumn{3}{|c|}{ Libration } \\
\hline $\begin{array}{c}298 \mathrm{~K} \\
\mathrm{nC}_{\mathrm{n}} \mathrm{H}_{2 \mathrm{n}+2} \\
\end{array}$ & $\begin{array}{l}\mathrm{A}_{\mathrm{ID}} \\
/ 10^{-1} \\
\end{array}$ & $\begin{array}{l}\tau \\
/ \mathrm{ps}\end{array}$ & $\begin{array}{c}\Omega_{\mathrm{I}} \\
/ \mathrm{THz} \\
\end{array}$ & $\begin{array}{l}A_{\mathrm{ID}} \\
/ 10^{-1} \\
\end{array}$ & $\begin{array}{l}\tau \\
/ \mathrm{ps} \\
\end{array}$ & $\begin{aligned} & \Omega_{\mathrm{I}} \\
& / \mathrm{THz} \\
&\end{aligned}$ & $\begin{array}{c}\mathrm{A}_{\mathrm{B}} \\
/ 10^{-1}\end{array}$ & $\begin{array}{l}\omega_{0} / 2 \pi \\
/ \mathrm{THz}\end{array}$ & $\begin{array}{l}\gamma / 2 \pi \\
/ \mathrm{THz}\end{array}$ & $\begin{array}{c}A_{B} \\
/ 10^{-1}\end{array}$ & $\begin{array}{l}\omega_{0} / 2 \pi \\
/ \mathrm{THz}\end{array}$ & $\begin{array}{l}\gamma / 2 \pi \\
/ \mathrm{THz}\end{array}$ \\
\hline 5 & 3.34 & 2.60 & 1.57 & 2.13 & 0.69 & 1.22 & 3.69 & 1.22 & 1.66 & 1.89 & 1.57 & 1.19 \\
\hline 6 & 5.23 & 5.23 & 1.70 & 2.19 & 1.12 & 1.21 & 3.20 & 1.21 & 1.79 & 1.75 & 1.70 & 1.33 \\
\hline 7 & 8.36 & 8.69 & 1.75 & 1.93 & 1.23 & 1.20 & 2.85 & 1.20 & 1.91 & 1.83 & 1.76 & 1.41 \\
\hline 8 & 11.0 & 13.9 & 1.82 & 1.78 & 1.41 & 1.22 & 2.50 & 1.22 & 1.97 & 1.82 & 1.82 & 1.46 \\
\hline 9 & 13.4 & 24.4 & 1.82 & 1.53 & 2.40 & 1.09 & 2.33 & 1.09 & 2.05 & 1.96 & 1.82 & 1.52 \\
\hline 10 & 15.9 & 43.6 & 1.80 & 1.57 & 4.07 & 1.01 & 2.06 & 1.01 & 2.11 & 1.85 & 1.80 & 1.52 \\
\hline 12 & 20.6 & 87.5 & 1.77 & 1.42 & 6.30 & 0.93 & 1.84 & 0.93 & 2.14 & 1.92 & 1.77 & 1.52 \\
\hline 14 & 23.5 & 162 & 1.78 & 1.65 & $9 \cdot 38$ & 0.88 & 1.71 & 0.88 & 2.17 & 1.85 & 1.78 & 1.57 \\
\hline 16 & 24.0 & 244 & 1.80 & 1.56 & 1.38 & 0.79 & 1.59 & 0.79 & 2.22 & 1.90 & 1.80 & 1.66 \\
\hline
\end{tabular}

Table $S_{3}$. Fit parameters for liquid methane at $95 \mathrm{~K}$. $\Omega_{\mathrm{I}}$ is fixed to the fast $-\beta \omega_{\mathrm{o}}$.

\begin{tabular}{|c|c|c|c|c|c|c|c|c|c|}
\hline \multirow[b]{2}{*}{$\mathrm{CH}_{4}$} & \multicolumn{3}{|c|}{$\beta$-relaxation } & \multicolumn{3}{|c|}{ Fast- $\beta$} & \multicolumn{3}{|c|}{ Bucaro-Litovitz } \\
\hline & $\begin{array}{l}A_{C C} \\
/ 10^{-1}\end{array}$ & $\begin{array}{c}\tau \\
/ \mathrm{ps}\end{array}$ & $\begin{array}{c}\Omega_{\mathrm{I}} \\
/ \mathrm{THz}\end{array}$ & $\begin{array}{c}A_{B} \\
/ 10^{-1}\end{array}$ & $\begin{array}{l}\omega_{\circ} / 2 \pi \\
/ \mathrm{THz}\end{array}$ & $\begin{array}{l}\gamma / 2 \pi \\
/ \mathrm{THz}\end{array}$ & $\begin{array}{c}\mathrm{A}_{\mathrm{BL}} \\
/ 10^{-4}\end{array}$ & $\begin{array}{c}\omega_{\mathrm{BL}} / 2 \pi \\
/ \mathrm{THz}\end{array}$ & \\
\hline & 0.46 & 0.76 & 1.66 & 1.76 & 1.66 & 1.29 & - & - & - \\
\hline & - & - & - & - & - & - & 2.60 & 0.95 & 1.05 \\
\hline
\end{tabular}


Table S4. Fit parameters for liquid propane for various temperatures. $\Omega_{\mathrm{I}}$ are fixed values; $\alpha$-relaxation $\Omega_{\mathrm{I}}$ is fixed to the libration $\omega_{\mathrm{G}}$ and $\beta$ relaxation $\Omega_{\mathrm{I}}$ is fixed to the fast $-\beta \omega_{\text {o }}$.

\begin{tabular}{|c|c|c|c|c|c|c|c|c|c|c|c|c|c|}
\hline \multirow[b]{2}{*}{$\begin{array}{l}\mathrm{T} \\
/ \mathrm{K}\end{array}$} & \multicolumn{3}{|c|}{$\alpha$-relaxation } & \multicolumn{4}{|c|}{$\beta$-relaxation } & \multicolumn{3}{|c|}{ Fast- $\beta$} & \multicolumn{3}{|c|}{ Libration } \\
\hline & $\begin{array}{l}A_{I D} \\
/ 10^{-1}\end{array}$ & $\begin{array}{c}\tau \\
/ \mathrm{ps}\end{array}$ & $\begin{array}{l}\Omega_{\mathrm{I}} \\
/ \mathrm{THz}\end{array}$ & $\begin{array}{l}A_{C C} \\
/ 10^{-1}\end{array}$ & $\begin{array}{l}\tau \\
/ \mathrm{ps} \\
\end{array}$ & $\begin{array}{c}\Omega_{\mathrm{I}} \\
/ \mathrm{THz}\end{array}$ & $\beta_{\mathrm{CC}}$ & $\begin{array}{c}A_{B} \\
/ 10^{-1}\end{array}$ & $\begin{array}{l}\omega_{\circ} / 2 \pi \\
/ \mathrm{THz}\end{array}$ & $\begin{array}{l}\gamma / 2 \pi \\
/ \mathrm{THz}\end{array}$ & $\begin{array}{c}A_{G} \\
/ 10^{-1}\end{array}$ & $\begin{array}{l}\omega_{\mathrm{G}} / 2 \pi \\
/ \mathrm{THz}\end{array}$ & $\begin{array}{l}\sigma_{\mathrm{G}} / 2 \pi \\
/ \mathrm{THz}\end{array}$ \\
\hline 77.7 & 1.80 & 25.9 & 1.58 & 2.09 & 1.54 & 1.74 & 0.72 & 1.33 & 1.74 & 2.08 & 1.19 & 1.58 & 1.53 \\
\hline 90 & 1.66 & 9.64 & 1.57 & 2.07 & 1.31 & 1.69 & 0.96 & 1.43 & 1.69 & 2.04 & 1.30 & 1.57 & 1.57 \\
\hline 130 & 1.42 & 6.16 & 1.49 & 1.99 & 0.77 & 1.59 & 1 & 1.70 & 1.59 & 1.92 & 1.15 & 1.49 & 1.58 \\
\hline 170 & 0.83 & 2.74 & 1.39 & 2.14 & 0.55 & 1.45 & 1 & 2.03 & 1.45 & 1.73 & 1.38 & 1.39 & 1.54 \\
\hline 210 & 0.38 & 1.93 & 1.24 & 2.04 & 0.47 & 1.41 & 1 & 2.28 & 1.41 & 1.59 & 1.61 & 1.24 & 1.54 \\
\hline
\end{tabular}

Table $S_{5}$. Fit parameters for the cycloalkanes at $25^{\circ} \mathbf{C} . \Omega_{\mathrm{I}}$ are fixed values; $\alpha$-relaxation $\Omega_{\mathrm{I}}$ is fixed to the libration $\omega_{\mathrm{G}}$ and $\beta$-relaxation $\Omega_{\mathrm{I}}$ is fixed to the fast $\beta \omega_{\circ}$.

\begin{tabular}{|c|c|c|c|c|c|c|c|c|c|c|c|c|c|}
\hline & \multicolumn{3}{|c|}{$\alpha$-relaxation } & \multicolumn{4}{|c|}{$\beta$-relaxation } & \multicolumn{3}{|c|}{ Fast- $\beta$} & \multicolumn{3}{|c|}{ Libration } \\
\hline $\begin{array}{c}298 \mathrm{~K} \\
\mathrm{cC}_{\mathrm{n}} \mathrm{H}_{2 \mathrm{n}}\end{array}$ & $\begin{array}{c}A_{I D} \\
/ 10^{-2}\end{array}$ & $\begin{array}{c}\tau \\
/ \mathrm{ps}\end{array}$ & $\begin{array}{l}\Omega_{\mathrm{I}} \\
/ \mathrm{THz} \\
\end{array}$ & $\begin{array}{l}A_{C C} \\
/ 10^{-2}\end{array}$ & $\begin{array}{c}\tau \\
/ \mathrm{ps}\end{array}$ & $\begin{array}{l}\Omega_{\mathrm{I}} \\
/ \mathrm{THz} \\
\end{array}$ & $\beta_{\mathrm{CC}}$ & $\begin{array}{c}\mathrm{A}_{\mathrm{B}} \\
/ 10^{-2}\end{array}$ & $\begin{array}{l}\omega_{0} / 2 \pi \\
/ \mathrm{THz}\end{array}$ & $\begin{array}{l}\gamma / 2 \pi \\
/ \mathrm{THz}\end{array}$ & $\begin{array}{c}\mathrm{A}_{\mathrm{G}} \\
/ 10^{-2}\end{array}$ & $\begin{array}{l}\omega_{\mathrm{G}} / 2 \pi \\
/ \mathrm{THz}\end{array}$ & $\begin{array}{l}\sigma_{\mathrm{G}} / 2 \pi \\
/ \mathrm{THz}\end{array}$ \\
\hline 5 & 3.55 & 0.50 & 0.95 & 7.88 & 0.89 & 1.23 & 1 & 28.8 & 1.23 & 1.26 & 11.0 & 0.95 & 1.60 \\
\hline 6 & 5.46 & 1.30 & 1.02 & 6.96 & 1.41 & 1.22 & 1 & 24.5 & 1.22 & 1.64 & 14.9 & 1.02 & 1.47 \\
\hline 7 & 10.8 & 6.52 & 1.10 & 13.9 & 1.55 & 1.22 & 1 & 22.7 & 1.22 & 2.13 & 14.6 & 1.10 & 1.30 \\
\hline 8 & 9.47 & 10.6 & 1.05 & 13.4 & 2.19 & 1.17 & 1 & 22.0 & 1.17 & 2.56 & 14.5 & 1.05 & 1.20 \\
\hline 10 & 32.8 & 47.9 & 0.97 & 28.4 & 4.57 & 1.04 & 0.84 & 19.0 & 1.04 & 2.50 & 13.1 & 0.97 & 1.17 \\
\hline
\end{tabular}


Table S6. Fit parameters for the 6-membered rings at $25^{\circ} \mathrm{C}$. $\Omega_{\mathrm{I}}$ are fixed values; $\alpha$-relaxation $\Omega_{\mathrm{I}}$ is fixed to the libration $\omega_{\mathrm{G}}$ and $\beta$-relaxation $\Omega_{\mathrm{I}}$ is fixed to the fast- $\beta \omega_{\circ}$.

\begin{tabular}{|c|c|c|c|c|c|c|c|c|c|c|c|c|}
\hline & \multicolumn{3}{|c|}{$\alpha$-relaxation } & \multicolumn{3}{|c|}{$\beta$-relaxation } & \multicolumn{3}{|c|}{ Fast- $\beta$} & \multicolumn{3}{|c|}{ Libration } \\
\hline $\begin{array}{c}298 \mathrm{~K} \\
\mathrm{C}_{6} \text { rings }\end{array}$ & $\begin{array}{c}\mathrm{A}_{\mathrm{ID}} \\
/ 10^{-2}\end{array}$ & $\begin{array}{c}\tau \\
/ \mathrm{ps}\end{array}$ & $\begin{array}{c}\Omega_{\mathrm{I}} \\
/ \mathrm{THz}\end{array}$ & $\begin{array}{l}\mathrm{A}_{\mathrm{ID}} \\
/ 10^{-2}\end{array}$ & $\begin{array}{l}\tau \\
/ \mathrm{ps}\end{array}$ & $\begin{array}{l}\Omega_{\mathrm{I}} \\
/ \mathrm{THz}\end{array}$ & $\begin{array}{c}A_{B} \\
/ 10^{-2}\end{array}$ & $\begin{array}{l}\omega_{0} / 2 \pi \\
/ \mathrm{THz}\end{array}$ & $\begin{array}{l}\gamma / 2 \pi \\
/ \mathrm{THz}\end{array}$ & $\begin{array}{c}\mathrm{A}_{\mathrm{G}} \\
/ 10^{-2}\end{array}$ & $\begin{array}{l}\omega_{\mathrm{G}} / 2 \pi \\
/ \mathrm{THz}\end{array}$ & $\begin{array}{l}\sigma_{\mathrm{G}} / 2 \pi \\
/ \mathrm{THz}\end{array}$ \\
\hline $\mathrm{C}_{6} \mathrm{H}_{12}$ & 5.46 & 1.30 & 1.02 & 6.96 & 1.41 & 1.22 & 24.5 & 1.22 & 1.64 & 14.9 & 1.02 & 1.47 \\
\hline $\mathrm{C}_{6} \mathrm{H}_{10}$ & 104 & 3.35 & 1.05 & 95.9 & 0.88 & 1.21 & 87.9 & 1.21 & 1.75 & 33.1 & 1.05 & 1.48 \\
\hline $1,4-\mathrm{C}_{6} \mathrm{H}_{8}$ & 290 & 2.88 & 1.22 & 201 & 0.76 & 1.20 & 160 & 1.20 & 1.85 & 64.8 & 1.22 & 1.62 \\
\hline $\mathrm{C}_{6} \mathrm{H}_{6}$ & 296 & 3.05 & 1.77 & 192 & 0.74 & 1.26 & 175 & 1.26 & 1.90 & 107 & 1.77 & 1.68 \\
\hline
\end{tabular}

\section{REFERENCES}

(1) Minhaeng, C.; Mei, D.; Norbert, F. S.; Graham, R. F.; Shaul, M. Off-Resonant Transient Birefringence in Liquids. J. Chem. Phys. 1993, 99, 2410-2428.

(2) Fecko, C. J.; Eaves, J. D.; Tokmakoff, A. Isotropic and Anisotropic Raman Scattering from Molecular Liquids Measured by Spatially Masked Optical Kerr Effect Spectroscopy. J. Chem. Phys. 2002, 117, 1139-1154.

(3) Buckingham, R. A. The Classical Equation of State of Gaseous Helium, Neon and Argon. Proc. R. Soc. A 1938, 168, 264-283.

(4) Lemmon, E.; McLinden, M.; Friend, D. Thermophysical Properties of Fluid Systems. In NIST Chemistry WebBook; Linstrom, P. J., Mallard, W. G., Eds; NIST Standard Reference Database Number 69; National Institute of Standards and Technology: Gaithersburg, MD, http://webbook.nist.gov, (retrieved September 20, 2019).

(5) Iglesias-Silva, G. A.; Guzmán-López, A.; Pérez-Durán, G.; Ramos-Estrada, M. Densities and Viscosities for Binary Liquid Mixtures of N-Undecane + 1-Propanol, + 1-Butanol, + 1-Pentanol, and + 1-Hexanol from 283.15 to $363.15 \mathrm{~K}$ at o.1 MPa. J. Chem. Eng. Data 2016, 61, 2682-2699.

(6) Fischer, J.; Weiss, A. Transport Properties of Liquids Vi. Viscosity, Excess Volumes, and Self-Diffusion of Nearly Athermal Mixtures. Ber. Bunsenges. Phys. Chem. 1986, 90, 1129-1141.

(7) Turton, D. A.; Wynne, K. Stokes-Einstein-Debye Failure in Molecular Orientational Diffusion: Exception or Rule? J. Phys. Chem. B 2014, 118, 4600-4604.

(8) Debye, P. Polar Molecules; The Chemical Catalog Company, inc.: New York, U.S.A., 1929.

(9) Doi, M. Rotational Relaxation Time of Rigid Rod-like Macromolecule in Concentrated Solution. J. Phys. (Paris) 1975, 36, 607-611.

(10) Neild, A.; Padding, J. T.; Yu, L.; Bhaduri, B.; Briels, W. J.; Ng, T. W. Translational and Rotational Coupling in Brownian Rods near a Solid Surface. Phys. Rev. E 2010, 82, 1-10.

(11) Perrin, F. Mouvement Brownien d'un Ellipsoïde (I). Dispersion Diélectrique Pour Des Molécules Ellipsoïdales. Le J. Phys. Radium 1934, 5, 497-511.

(12) Hu, C. M.; Zwanzig, R. Rotational Friction Coefficients for Spheroids with the Slipping Boundary Condition. J. Chem. Phys. 1974, 60, 4354-4357.

(13) Einstein, A. Über Die von Der Molekularkinetischen Theorie Der Wärme Geforderte Bewegung von in Ruhenden Flüssigkeiten Suspendierten Teilchen. Ann. Phys. (Leipzig) 1905, 322, 549-560. 